\title{
IMAGE RECONSTRUCTION FOR MAGNETIC PARTICLE IMAGING USING AN AUGMENTED LAGRANGIAN METHOD
}

\author{
Serhat Ilbey ${ }^{1}$, Can Barış Top ${ }^{1}$, Tolga Çukur ${ }^{2,3}$, Emine Ulku Saritas ${ }^{2,3}$, H. Emre Güven ${ }^{1}$ \\ ${ }^{1}$ Advanced Sensing Research Program Department, ASELSAN A. Ş., Turkey \\ ${ }^{2}$ Department of Electrical and Electronics Engineering, Bilkent University, Ankara, Turkey \\ ${ }^{3}$ National Magnetic Resonance Research Center (UMRAM), Bilkent University, Ankara, Turkey
}

\begin{abstract}
Magnetic particle imaging (MPI) is a relatively new imaging modality that images the spatial distribution of superparamagnetic iron oxide nanoparticles administered to the body. In this study, we use a new method based on Alternating Direction Method of Multipliers (a subset of Augmented Lagrangian Methods, ADMM) with total variation and $l_{1}$ norm minimization, to reconstruct MPI images. We demonstrate this method on data simulated for a field free line MPI system, and compare its performance against the conventional Algebraic Reconstruction Technique. The ADMM improves image quality as indicated by a higher structural similarity, for low signal-to-noise ratio datasets, and it significantly reduces computation time.
\end{abstract}

Index Terms - Magnetic particle imaging, field free line, augmented Lagrangian method, algebraic reconstruction technique, alternating direction method of multipliers.

\section{INTRODUCTION}

Magnetic Particle Imaging (MPI) is a relatively new imaging modality in which superparamagnetic iron oxide (SPIO) nanoparticles are introduced inside the body, and their spatial distribution (density) is imaged [1,2]. The applications of MPI include -but are not limited to- angiography, stem cell tracking, and tumor imaging. In MPI, a static magnetic field (selection field) is applied to create a field free region (FFR). This magnetic field can be generated using a pair of coils with opposite polarity. The SPIOs inside the FFR respond to an alternating magnetic field (drive field) by changing their magnetization direction via Brownian and Néel relaxation mechanisms. Outside the FFR, the SPIOs are saturated with negligible response to the applied drive field. Since the magnetization curve of the SPIOs is nonlinear, the nanoparticles within the FFR yield a magnetization response that contains harmonics of the applied drive field frequency. This response is detected via an inductive receive coil, where signal amplitude depends on the number of SPIOs inside the FFR. The FFR is then swept through the entire imaging volume to generate a three-dimensional image. The resolution of this image depends on the magnetic field gradient in the FFR, magnetization properties of the SPIOs, and the signal-to-noise ratio (SNR) of the received signal [3, 4].

MPI images can be reconstructed either using a system matrix approach that involves a separate calibration procedure [1-5], or alternatively in the image domain (or xspace) based on several assumptions on the SPIO properties and the magnetic field [6]. In the calibration procedure of the system matrix method, a small SPIO sample is placed at a specific voxel location while the entire field-of-view (FOV) is scanned. This procedure is repeated for all voxel locations within the FOV. The responses measured across all voxel locations are then saved as the system matrix. Next, the actual object of interest is imaged, and a linear system of equations (LSE) is formed using the system matrix and the response of the object. The unknown SPIO distribution is reconstructed by solving this LSE. Typically, this inverse problem is solved via iterative regularized methods such as the Kaczmarz method, also known as Algebraic Reconstruction Technique (ART) [5]. Nonnegative fused lasso model, which minimizes the total variation and $l_{l}$ norm solution, is reported to perform better for preserving edge discontinuities in the image [7].

In MPI, the FFR is typically characterized as a small ellipsoidal volume, which is called a Field Free Point (FFP) [1-7]. As the received signal is directly related to the number of particles in the FFR, field free line (FFL) imaging is proposed to increase sensitivity via using a larger FFR volume [8]. The conventional reconstruction method in FFL MPI is based on projection imaging, which uses the time domain data to perform the reconstruction in the image domain [9-11].

In this paper, we use an FFL-type MPI scanner and the system-matrix reconstruction method to image a $2 \mathrm{D}$ numerical vessel phantom. We use two different approaches to solve the convex problem described by the LSE. In the first one, we use a formulation minimizing total variation (TV) and $l_{l}$ norm [12], similar to the nonnegative fused lasso model [7]. In the second one, we solve a regularized least square problem formulation, which is considered as the state-of-theart reconstruction method in MPI [7]. We compare these two formulations by using a hybrid Alternating Direction Method 
of Multipliers (ADMM) solution for the former, and ART solution for the latter, in terms of image quality and convergence time for various SNR values.

\section{METHODS}

\subsection{MPI Configuration}

An ideal FFL (homogeneous, straight) with $2 \mathrm{~T} / \mathrm{m}$ selection field gradient was modeled numerically and rotated inside a $48 \mathrm{~mm}$ x $48 \mathrm{~mm}$ FOV with 3 degree steps for 180 degrees. To translate the FFL, a $60 \mathrm{mT}$ amplitude $25 \mathrm{kHz}$ drive field was used. SPIO diameter was assumed to be $25 \mathrm{~nm}$. Imaging domain was discretized with $300 \mu \mathrm{m} \times 300 \mu \mathrm{m}$ pixel size. A drive field was applied to move the FFL in the direction orthogonal to it. Then, the received signal at each rotation angle was calculated using $[7,13]$ :

$$
R_{x}(t)=-\frac{\partial}{\partial t} \mu_{0} \iint \rho(x, y) L(\beta|\boldsymbol{H}|) q_{r} d x d y
$$

where $\rho(x, y)$ is the magnetic particle distribution inside the FOV. $\mu_{0}(H / m)$ is the magnetic permeability of free space. $\mathrm{L}$ is the Langevin function. $\mathrm{H}$ is the time dependent magnetic field inside the FOV. $q_{r}$ is the fraction of the magnetic field sensed by the receiver coils. $\beta=\mu_{0} \mathrm{M}_{\mathrm{sat}} V / \mathrm{k}_{\mathrm{B}} \mathrm{T}_{\mathrm{p}} . \mathrm{M}_{\mathrm{sat}}$ is the saturation magnetization $\left(0.6 \mathrm{~T} / \mu_{0}\right), \mathrm{V}\left(\mathrm{m}^{3}\right)$ is the volume of a single magnetic particle, $\mathrm{k}_{\mathrm{B}}$ is the Boltzmann constant $\left(1.38 \times 10^{-23}\right), \mathrm{T}$ is the particle temperature $(305 \mathrm{~K})$. A 5-cycle drive field was applied at each FFL angle. The received signal was sampled at $10 \mathrm{MHz}$. Additive white Gaussian noise was added to the data to model the effect of noise at SNR values of $30 \mathrm{~dB}, 20 \mathrm{~dB}$, and $10 \mathrm{~dB}$.

The system matrix was formed by sweeping a singlepixel object across the entire FOV, and measuring the responses at each pixel location. Next, the numerical vessel phantom shown in Fig. 1 was used in the simulation of the measurement process. Frequency components up to 50 harmonics (discarding the first harmonic) were used for image reconstruction. All simulations were done using MATLAB on an Intel ${ }^{\circledR}$ Xeon $^{\circledR}$ workstation with E5-2650, 2 of $2 \mathrm{GHz}$ CPUs and $64 \mathrm{~GB}$ RAM.

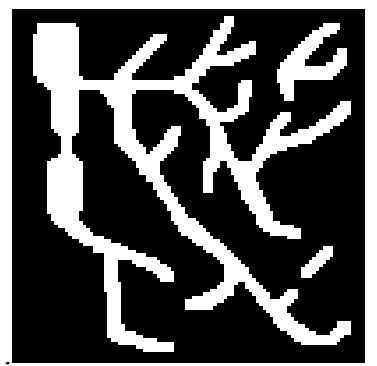

Figure 1. Numerical vessel phantom used in the study

\subsection{Reconstruction using ART}

For ART reconstruction, the following regularized leastsquares problem was solved [5]:

$$
\operatorname{argmin}_{\widehat{x}}\|\widehat{A} \widehat{x}-\boldsymbol{b}\|^{2}+\lambda\|\widehat{x}\|^{2}
$$

where $\widehat{\boldsymbol{A}}=\left(\begin{array}{ll}\boldsymbol{A} & \alpha \boldsymbol{I}\end{array}\right)$, and $\widehat{\boldsymbol{x}}=\left(\begin{array}{l}\boldsymbol{x} \\ 0\end{array}\right) . \boldsymbol{A}$ is the system matrix in frequency domain, $\boldsymbol{b}$ is the measurement vector, and $\boldsymbol{x}$ is the image vector. The Kaczmarz method solves the convex problem by projecting the solution on the rows of $\widehat{A}$ matrix iteratively:

$$
\boldsymbol{x}^{k+1}=\boldsymbol{x}^{k}+\lambda \frac{b_{i}-\left\langle\boldsymbol{a}_{i}, \boldsymbol{x}^{k}\right\rangle}{\left\|\boldsymbol{a}_{i}\right\|^{2}} \boldsymbol{a}_{i}
$$

where $\mathrm{k}$ is the iteration step; $\boldsymbol{a}_{i}$ and $b_{i}$, are the $i^{\text {th }}$ row of $\widehat{\boldsymbol{A}}$ matrix and $i^{\text {th }}$ component of the measurement vector $\boldsymbol{b}$; and $\lambda$ is a scalar weighting coefficient.

\subsection{Reconstruction using ADMM}

For ADMM reconstruction, a weighted sum of the total variation and the $l_{l}$ norm of the image was minimized. We consider this suitable for MPI images, as they are typically sparse by nature [6] and they tend to have block-wise contiguous features that motivate the use of total variation. For example, in an angiographic image, the signal originates solely from within the blood vessels. In addition, we applied a nonnegativity constraint. The convex optimization problem was formulated as follows:

$$
\begin{gathered}
\operatorname{argmin}_{\boldsymbol{x}} \alpha_{1}\|\boldsymbol{x}\|_{1}+\alpha_{2} T V(\boldsymbol{x}) \\
\text { subject to }\|\boldsymbol{A} \boldsymbol{x}-\boldsymbol{b}\|<\epsilon, \boldsymbol{x}_{i} \geq 0 \text { for } i=\{1, \ldots, N\}
\end{gathered}
$$

where $\mathrm{N}$ is the total number of pixels, which was $160 \times 160=$ 25600 in our experiment. This problem was solved using a hybrid ADMM algorithm [12].

\section{RESULTS}

The reconstruction results are displayed in Fig. 2. Figs. 3-5 show the corresponding convergence curves, i.e., structural similarity index measure (SSIM) and normalized root mean square error (NRMSE) as a function of computation time (measured with cputime function in MATLAB). SSIM is calculated with ssim function in MATLAB Image Processing Toolbox (IPT) whereas NRMSE is calculated with the following formula:

$$
\text { nrmse }=\sqrt{\operatorname{immse}(x, r e f)} /(\max (x)-\min (x))
$$

Here immse is a function in MATLAB IPT, max and min functions stand for maximum and minimum element of $\mathrm{x}$, respectively. The following cases are reported: no noise, $30 \mathrm{~dB}$ SNR, $20 \mathrm{~dB}$ SNR and $10 \mathrm{~dB}$ SNR. 

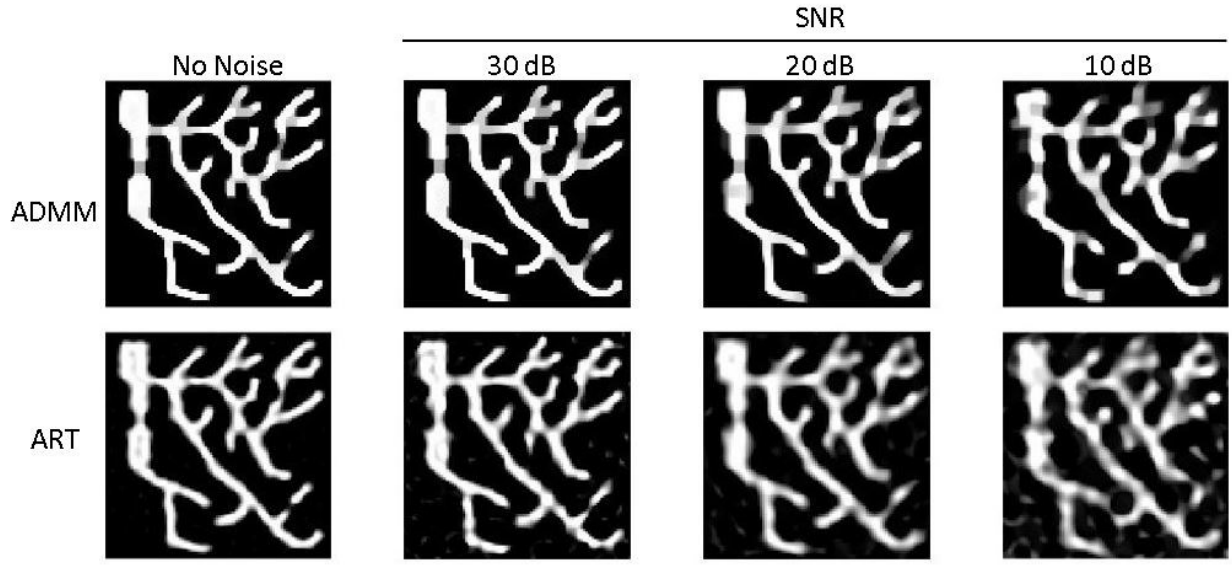

Figure 2. Reconstructed images using the ART and ADMM methods for various SNR values.

For a fixed computation time, SSIM of the reconstructed images is much higher with the ADMM method relative to the ART method, especially at lower SNR values. Also, visual inspection suggests that ADMM images have improved contrast and tissue depiction. This observation is confirmed by quantitative contrast measurements (calculated using the method given in [14]) for the reconstructions in Fig. 2 that are listed in Table 1. In the initial iteration steps, NRMSE of ART is lower than ADMM. Nevertheless, as the algorithms converge, ADMM outperforms ART in terms of NSMRE metric as well.

Table 1. Comparison of image contrast for the reconstructed images in Figure 2.

\begin{tabular}{|l|c|c|c|c|}
\cline { 2 - 5 } \multicolumn{1}{c|}{} & Noiseless & $\mathbf{3 0} \mathbf{~ d B}$ & $\mathbf{2 0} \mathbf{~ d B}$ & $\mathbf{1 0} \mathbf{~ d B}$ \\
\hline ADMM & 1.72 & 1.68 & 1.64 & 1.62 \\
\hline ART & 1.60 & 1.57 & 1.37 & 1.21 \\
\hline
\end{tabular}

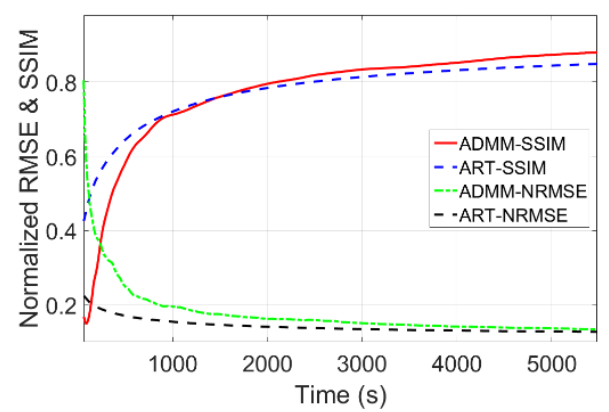

Figure 3. SSIM and NRMSE as a function of cumulative computation time for the noise-free case.

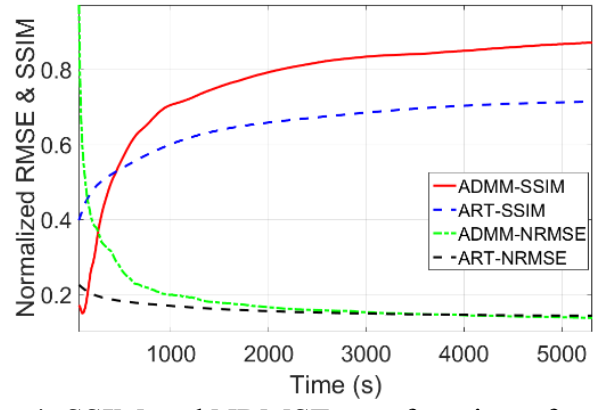

Figure 4. SSIM and NRMSE as a function of cumulative computation time at $30 \mathrm{~dB}$ SNR

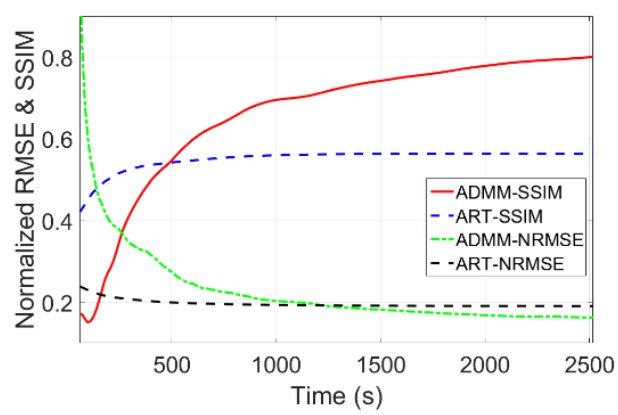

Figure 5. SSIM and NRMSE as a function of cumulative computation time at $20 \mathrm{~dB}$ SNR

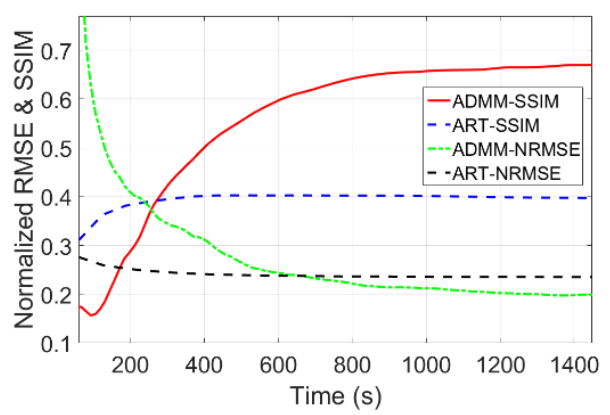

Figure 6. SSIM and NRMSE as a function of cumulative computation time at $10 \mathrm{~dB}$ SNR 


\section{DISCUSSION}

ADMM outperforms ART reconstruction in terms of SSIM levels, particularly at lower SNRs. When the noise level is increased, it is observed that the gap between SSIM convergence levels of ADMM and ART increases in favor of ADMM.

It should be noted that a relatively high drive field amplitude was utilized in this study to cover the whole FOV in a single scan. It was also assumed that a high receiver bandwidth could be used, which may not be easy to achieve in practice. One practical alternative is to divide the FOV into smaller FOVs to lower the drive field amplitude, which was shown to increase resolution with small number of received harmonics [15]. In fact, such an approach may be necessary to avoid nerve stimulation or prevent over-heating of the tissue for human imaging applications [16].

\section{CONCLUSION}

In this study, two iterative reconstruction methods are analyzed for the field free line magnetic particle imaging in terms of image quality and reconstruction time. The results show that hybrid Alternating Direction Method of Multipliers (ADMM) method converges much faster than the Kaczmarz method (ART). Moreover, ADMM results in significantly higher structural similarity index measure levels at low signal-to-noise ratio. Using low field amplitude yielding a high resolution with smaller bandwidth, reconstruction speed may be the determining factor for the choice of reconstruction method. In this case, the ADMM method will likely be preferable to the ART reconstruction.

\section{ACKNOWLEDGMENT}

The authors would like to thank Alper Güngör for providing the MATLAB implementation of the algorithm in [12].

\section{REFERENCES}

[1] B. Gleich and J. Weizenecker. Tomographic imaging using the nonlinear response of magnetic particles. Nature, 435(7046):1217-1217, 2005.

[2] EU Saritas, PW Goodwill, LR Croft, JJ Konkle, K Lu, B Zheng and SM Conolly. "Magnetic Particle Imaging (MPI) for NMR and MRI Researchers". J Magn Reson, 229:116-126, 2013.

[3] J. Weizenecker, J. Borgert, and B. Gleich, "A simulation study on the resolution and sensitivity of magnetic particle imaging," Phys. Med. Biol., vol. 52, pp. 6363-6374, 2007.

[4] Knopp, T., Biederer, S., Sattel, T.F., Erbe, M., Buzug, T.M., Prediction of the spatial resolution of magnetic particle imaging using the modulation transfer function of the imaging process. IEEE Trans. Med. Imaging 30(6), 1284-1292 (2011).
[5] Knopp, T., Rahmer, J., Sattel, T.F., Biederer, S., Weizenecker, J., Gleich, B., Borgert, J., Buzug, T.M.: Weighted iterative reconstruction for magnetic particle imaging. Phys. Med. Biol. 55(8), 1577-1589 (2010).

[6] P. Godwill and S. Conolly, "Multi-dimensional x-space magnetic prticle imaging," IEEE Trans. Med. Imag., vol. 30, no. 9, pp. 1581-1590, Sep. 2011.

[7] M. Storath et al., "Edge preserving and noise reducing reconstruction for magnetic particle imaging," IEEE Transactions on Medical Imaging, vol. PP, no. 99, pp. 1-1, 2016.

[8] Weizenecker, J., Gleich, B., Borgert, J.: Magnetic particle imaging using a field free line. J. Phys. D 41(10), 3pp (2008).

[9] T. Knopp, M. Erbe, T. F. Sattel, S. Biederer, and T. M. Buzug., A Fourier slice theorem for magnetic particle imaging using a field free line. Inverse Problems, 27(9):095004, 2011.

[10] J. J. Konkle, P. W. Goodwill, O. M. Carrasco-Zevallos and S. M. Conolly, "Projection Reconstruction Magnetic Particle Imaging," in IEEE Transactions on Medical Imaging, vol. 32, no. 2, pp. 338-347, Feb. 2013.

[11] K. Bente, M. Weber, M. Graeser, T. F. Sattel, M. Erbe, and T. M. Buzug, "Electronic field free line rotation and relaxation deconvolution in magnetic particle imaging," IEEE Trans Med Imaging, vol. 34, no. 2, pp. 644-651, Feb. 2015.

[12] H. E. Guven, A. Gungor, and M. Cetin, "An Augmented Lagrangian Method for Complex-Valued Compressed SAR Imaging," IEEE Transactions on Computational Imaging, vol. 2, no. 3, pp. 235-250, Sep. 2016.

[13] P. W. Goodwill, J. J. Konkle, B. Zheng, E. U. Saritas, and S. M. Conolly, "Projection x-space magnetic particle imaging," IEEE Trans.Med. Imag., vol. 31, no. 5, pp. 1076-1085, May 2012.

[14] Martorella, M., F. Berizzi, and B. Haywood. "Contrast maximisation based technique for 2-D ISAR autofocusing." IEE Proceedings-Radar, Sonar and Navigation 152.4 (2005): 253-262.

[15] L. R. Croft et al., "Low drive field amplitude for improved image resolution in magnetic particle imaging," Medical Physics, vol. 43, no. 1, pp. 424-435, Jan. 2016.

[16] E.U. Saritas, PW Goodwill, GZ Zhang, SM Conolly. "Magnetostimulation Limits in Magnetic Particle Imaging". IEEE Trans Med Imag, 32(9):1600-1610, 2013 